Portland State University

PDXScholar

$12-2021$

\title{
Testing for Cognitive Deficits after Mild Traumatic Brain Injury using the Automated Neuropsychological Assessment Metric and Dual- Task Assessments
}

Jasmin Mabry

Follow this and additional works at: https://pdxscholar.library.pdx.edu/honorstheses

Part of the Rehabilitation and Therapy Commons Let us know how access to this document benefits you.

\section{Recommended Citation}

Mabry, Jasmin, "Testing for Cognitive Deficits after Mild Traumatic Brain Injury using the Automated Neuropsychological Assessment Metric and Dual-Task Assessments" (2021). University Honors Theses. Paper 1160.

https://doi.org/10.15760/honors.1183

This Thesis is brought to you for free and open access. It has been accepted for inclusion in University Honors Theses by an authorized administrator of PDXScholar. Please contact us if we can make this document more accessible: pdxscholar@pdx.edu. 
Testing for Cognitive Deficits after Mild Traumatic Brain Injury using the Automated Neuropsychological Assessment Metric and Dual-Task Assessments

by

Jasmin Mabry

An undergraduate honors thesis submitted in partial fulfillment of the requirements for the degree of

Bachelor of Science

University Honors

and

Psychology

Thesis Committee:

Laurie A. King, Ph.D., PT, MCR - Primary Advisor

Kody R. Campbell, Ph.D. - Co-advisor

Portland State University 


\begin{abstract}
Introduction: Cognitive function after mild traumatic brain injury (mTBI) is traditionally assessed using a seated computerized exam. Dual task (DT) gait assessments are also increasingly employed in the assessment of motor-cognitive function after mTBI. Cognitive deficits are common following mTBI, and it is unclear when these cognitive symptoms subside. Little is known about DT gait and neurocognitive performance in an older and more chronically affected ( $>3$ months post-injury) population. Herein, we compared people with chronic mTBI and subacute mTBI to healthy controls on DT gait and neurocognitive performance and explored the relationship between cognitive deficits, the method of cognitive evaluation, and time since injury. Methods: A total of 47 subjects with chronic mTBI ( $>3$ months post injury), 47 subjects with sub-acute mTBI (2-12 weeks post injury; $<3$ months) and 59 age and sex matched healthy control subjects were used for analysis. Subjects completed a traditional computerized neurocognitive test - the Automated Neuropsychological Assessment Metrics (ANAM), and a motor-cognitive DT test which assessed both motor performance with a cognitive task as well as the cognitive task accuracy. The DT test consisted of subjects walking 13 meters while performing a cognitive auditory Stroop task. During these trials, subjects wore inertial measurement units that collected and calculated gait metrics. Auditory Stroop task accuracy was also recorded. Mann Whitney $U$ tests and pairwise comparisons were used to evaluate group differences on the ANAM cognitive composite score \& subcategory scores, as well as the DT cognitive accuracy, and DT gait speed.
\end{abstract}

Results: The ANAM composite score indicated that the healthy control populations significantly outperformed the subacute and chronic mTBI populations, however there was no significant difference between the mean subacute and chronic scores. In both ST and DT cognitive task accuracy, the subacute mTBI group performance was significantly better than the chronic population, and the healthy control population significantly outperformed the chronic group during the ST activity. Regardless of task, the chronic mTBI population also suffered in gait function.

Discussion: For people with chronic mTBI, cognitive and gait deficits may still be present months after the initial injury. These deficits can be detected using traditional neurocognitive evaluations and through DT gait assessments. The ANAM is sufficiently accurate at assessing cognitive deficits in individuals with mTBI, and the DT walking and auditory Stroop task's gait portion may indicate chronic cognitive impairment due to mTBI. 


\section{Acknowledgments}

First and foremost, I would like to thank my primary investigator and co-thesis advisor Dr. Laurie King for providing me with the resources and guidance needed to complete this project. As an undergraduate BUILD EXITO intern in the Balance Disorders Laboratory, I have never felt anything but supported and encouraged, and I am eternally grateful to have had the opportunity to work in this environment. I would also like to give a huge thank you to my mentor and co-thesis advisor Dr. Kody Campbell for acting as a pillar to my research education and guiding me through the process of writing this paper with infinite kindness and patience. Despite the unusual circumstances of the past couple of years, with COVID-19 and welcoming a new addition to your family, your support has never once wavered. I would also like to extend a warm thank you to everyone else in the Balance Disorders Laboratory. I could not have completed this project without your unlimited advice, generosity, and insurmountable expertise. Next, I would like to acknowledge the role of BUILD EXITO, without whom this project would not have been possible. Thank you for supporting not only me, but everyone in the program on their pathway to becoming scientific researchers. BUILD EXITO has not only provided me with endless resources, they have also provided me with transformative connections, such as my career mentor, Dr. Bill Griesar. Finally, I would like to thank my mother for being my biggest supporter. With English being your second language, it has never felt fair to me that your writing and grammar skills are infinitely superior to mine, but I have no right to complain when I get a mother and an editor in one. You truly are the wind beneath my wings. 


\section{Funding}

Research reported in this publication was supported by the by the Assistant Secretary of Defense for Health Affairs under the Award Numbers W81XWH-15-0620 and W81XWH-17-1-0424 and the National Institutes of Health Common Fund and Office of Scientific Workforce Diversity under three awards UL1GM118964, RL5GM118963, and TL4GM118965, administered by the National Institute of General Medical Sciences. Opinions, interpretations, conclusions, and recommendations are those of the author and are not necessarily endorsed by the US Department of Defense or the National Institutes of Health.

\section{Ethics Approval and Consent to Participate}

All participants completed written informed consent prior to testing. An investigator or research assistant verbally described the consent form and allowed time for participants to thoroughly read through the consent form followed by signing and dating the form. All protocols have been approved by the Oregon Health and Science (OHSU) and VA Portland Health Care System (VAPORHCS) Institutional Review Board (IRB).

\section{Declaration of Conflicting Interests}

I have no conflict of interest to report. 


\section{Table of Contents}

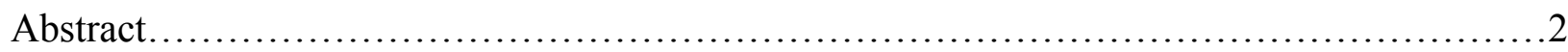

Acknowledgements...............................................................

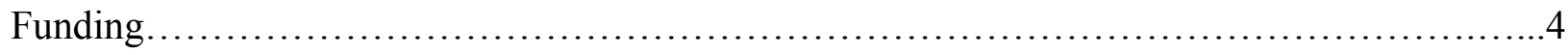

Ethics Approval and Consent to Participate..........................................

Declaration of Conflicting Interests............................................... 4

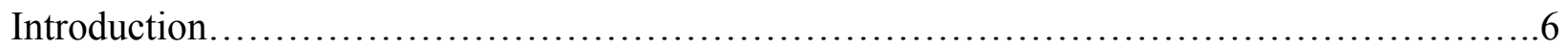

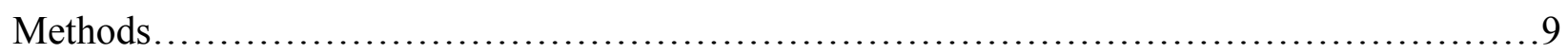

Participants...........................................................

Protocol and Data Collection..............................................11

Statistical Analysis......................................................14

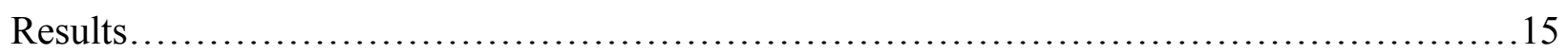

Traditional Neuropsychological Testing Between Group Differences.................15

Single Task and Dual Task Test Cognitive Group Differences......................17

Gait................................................................. 18

Discussion................................................................... 21

Limitations ............................................................. 24

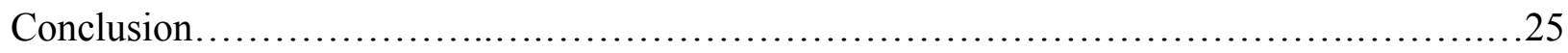

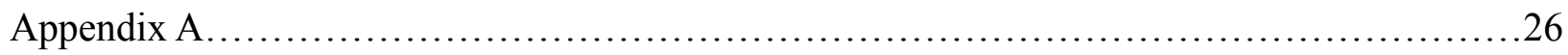

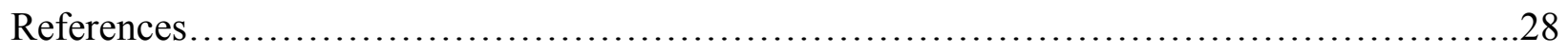




\section{Introduction}

Mild traumatic brain injury (mTBI) causes a myriad of symptoms, including: cognitive and emotional disruptions, headaches, general neurological deficits, balance impairments, behavioral changes, and sleep interference (McCrory et al., 2017). Of these symptoms, cognitive deficits are difficult to evaluate in a clinical setting due to the lack of overt neurological symptoms (Avedesian et al., 2021). However, neurological impairments can often persist after all other symptoms have subsided, and require sensitive measurement tools for appropriate management (Miotto et al., 2010).

From a clinical symptom perspective, patients with mTBI typically recover within the first month of injury (Broglio et al., 2021 \& Nelson et al., 2019). New research has revealed, however, that physiological and neurobiological recovery time may outlast current clinical measures of recovery time (Kamins et al., 2017, \& Porter, S.J. \& Johnson, D.E., 2020). This means that an individual can be clinically cleared for returning to their activities (i.e. work, school, sport, active duty service) before they are fully recovered. The consequences of returning prematurely to high risk activities could be the development of chronic symptoms such as ongoing cognitive impairments, or emotional disruption (such as depression) (Maruta et al., 2016). Additional consequences of returning to activity before full physiological recovery include chronic memory and concentration deficits, chronic migraines, and persistent balance impairments, which makes individuals more susceptible to suffering another mTBI (Recovery | Concussion | Traumatic Brain Injury | CDC Injury Center, 2020). For this reason, accurate and sensitive assessment measures of mTBI-related deficits are important for positive long-term outcomes for patients. 
There is conflicting evidence on if the various cognitive domains that are typically assessed remain impaired chronically ( $>3$ months) when assessed using different cognitive assessments (Dean \& Sterr, 2013). These domains include attention and concentration, working memory, mental flexibility, spatial processing, cognitive processing efficiency, and memory recall. The prevailing view seems to be that most cognitive functioning is impaired following mTBI, but improves throughout the first month of injury and is back to normal by 3 months post injury (Karr et al., 2014, Kwok et al., 2008, \& Levin et al.,1987, 1991). Nevertheless, there has been a recent shift in that perspective as a number of studies have shown that at least half of individuals with mTBI display long term cognitive impairment (McInnes et al., 2017). Seated comprehensive neuropsychological exams and motor-cognitive assessments are beginning to provide evidence of longer-term deficits in cognitive functioning.

One post-mTBI cognitive assessment measure is the Automated Neuropsychological Assessment Metric (ANAM). The ANAM was originally developed for use in a military setting after service members received head injuries from training or during deployed active combat settings, but has since become integrated into mTBI assessment both in research and in civilian clinical settings (Reeves et al., 2007). It consists of a battery of computerized tests designed to assess attention and concentration, working memory, mental flexibility, spatial processing, cognitive processing efficiency, memory recall, mood, fatigue level, and psychomotor performance (Reeves et al., 2007, p. 1-2). However, a seated cognitive test like the ANAM might not accurately reflect the function required of an mTBI patient in everyday life due to its inability to evaluate cognitive skills in conjunction with motor tasks such as walking. The external validity (the extent to which test results are generalizable to real world situations) of this form of testing 
is therefore questionable (Czerniak et al., 2020). Additionally, some evidence suggests that the test-retest reliability of the ANAM does not have adequate longevity to assess chronic mTBI patients, asserting that the results only maintain temporal stability within the first 2 weeks out from injury (Coldren et al., 2012 \& Dretsch et al., 2015).

Dual-task (DT) measures of cognitive and motor function may be able to uncover cognitive deficits in post mTBI populations with a higher sensitivity compared to seated, traditional neurocognitive computerized tests. In fact, when compared to motor-cognitive DT measures, neuropsychological tests have shown normal scores where DT tests show poor performance in acute, subacute, and chronic mTBI populations (Kleiner et al., 2018). This may be because DT assessments involve testing the cognitive ability of attentional allocation. Attention is a limited resource and when attention is divided between two tasks, performance in either one or both of the tasks will suffer (Plummer at al., 2015 \& Lee et al., 2012). Importantly, executive components of attention can be impaired and persist following mTBI (Howell et al., 2013, McCrea et al., 2003 Plummer et al., 2015). Although traditional cognitive tests like the ANAM measure attention similarly to DT activities, the test is conducted while sitting in a quiet room with no other distractions. This begs the question of whether it tests attention in a way that is similar to realworld scenarios.

The motor aspect of DT activities may also allow for more comprehensive assessments due to some evidence suggesting impaired gait performance and more cognitive errors in mTBI populations when undergoing DT assessments. These gait deficits are present despite normal scores on neuropsychological tests (Kleiner et al., 2018). Additionally, individuals with mTBI have been shown to walk and turn slower on average than healthy controls (Martini et al., 2021 
\& Büttner et al. 2020). This is especially prevalent in chronic mTBI populations, who typically exhibit altered gait and worse gait performance, particularly when undergoing DT activities. This further suggests that DT assessments may be capable of uncovering cognitive deficits in relation to gait that seated neurocognitive tests are not assessing. Given these findings, clinicians who are using seated neurocognitive tests (the standard practice) may be missing persistent neurological shortfalls in their evaluations of mTBI patients, and clearing them as healthy when they are not fully recovered.

The purpose of this thesis was to: 1) examine differences in cognitive abilities between healthy controls, people with subacute mTBI symptoms ( 2 to 12 weeks), and chronic symptoms ( $>12$ weeks), measured using traditional standardized computer cognitive testing (ANAM), and 2) examine differences in DT motor-cognitive function between healthy controls, subacute mTBI, and chronic mTBI persons. It was hypothesized that there would be differences in cognitive function between healthy controls and the mTBI groups, and that all groups would demonstrate gait differences during dual task assessments but there would be larger differences for the mTBI groups.

\section{Methods}

\section{Participants}

This thesis was a secondary data analysis on data collected from two separate studies that measured neurocognitive and DT motor-cognitive performance on healthy controls and people with sub-acute $(2-12$ week; $<3$ months $)$ and chronic $(>3$ months $)$ mTBI. The chronic population included $47 \mathrm{mTBI}$ and 59 healthy control subjects (NCT02748109) and the sub-acute mTBI 
population included data collected from 47 participants (NCT03479541). All participants were age and sex matched in order to account for potential confounding demographic variables.

Participant recruitment took place in the greater Portland metropolitan area through Oregon Health and Science University (OHSU) and local clinics. Common inclusion criteria for the mTBI populations included 1) being between 18 - 60 years old, 2) having an mTBI diagnosed or confirmed by a physician using Veterans Affairs (VA)/Department of Defense criteria, and 3) no more than minimal cognitive impairment (a score of $\leq 8$ on the Short Blessed Test). Further inclusion criteria for the sub-acute population included participants being within 2 to 12 weeks post-mTBI, exhibiting a graded symptom checklist total symptom severity score of $>15$, and endorsing any symptoms of headache, nausea, dizziness, blurred vision, or balance problems from the Sport Concussion Assessment Tool version 5. For the chronic population, participants had to be $>3$ months post mTBI with non-resolving, self-reported balance complaints. Common exclusion criteria for both healthy control and mTBI populations included 1) a previous or current musculoskeletal injury, surgery, medication, or neurological illness that would influence balance, 2) a reported substance-abuse in the past month, according to DSM-V classifications, 3) reporting or displaying significant pain, 4) a history of pathological symptoms that affect the musculoskeletal, peripheral vestibular, or ocular motor systems and, 5) being unable to refrain from using balance impairing medications for 24 hours prior to testing sessions. Lastly for the healthy control group, participants were excluded if they had a brain injury within the last year. Each study was approved by the Joint Institutional Review Board Committee of OHSU and VA Portland Health Care System. 
Table 1: Participant demographics for healthy control, subacute mild traumatic brain injury (mTBI), and chronic mTBI groups.

\begin{tabular}{lccc}
\hline & $\begin{array}{c}\text { Healthy Controls } \\
(\mathbf{n}=\mathbf{5 9})\end{array}$ & $\begin{array}{c}\text { Subacute mTBI } \\
(\mathbf{n}=\mathbf{4 7})\end{array}$ & $\begin{array}{c}\text { Chronic mTBI } \\
(\mathbf{n}=\mathbf{4 7})\end{array}$ \\
\hline Age (years) & $37.22(12.83)$ & $37.25(10.01)$ & $37.56(10.48)$ \\
Height (cm) & $171.36(9.78)$ & $169.67(8.23)$ & $170.14(9.62)$ \\
Mass (kg) & $75.36(19.38)$ & $70.08(12.05)$ & $80.44(20.12)$ \\
Body Mass Index (kg/m²) & $25.43(5.02)$ & $24.34(3.91)$ & $27.60(5.72)$ \\
Sex (M/F) & $23 \mathrm{M} / 36 \mathrm{~F}$ & $13 \mathrm{M} / 34 \mathrm{~F}$ & $13 \mathrm{M} / 34 \mathrm{~F}$ \\
NSI Total Score (out of 88) & $3.93(4.06)$ & $40.78(13.35)$ & $36.30(16.09)$ \\
Days Since Injury & & $36(24,56)$ & $426(191,1023)$ \\
Injury Mechanism & & & $0(0 \%)$ \\
Bike & & $3(6.4 \%)$ & $1(2.2 \%)$ \\
Blast & & $0(0 \%)$ & $6(13 \%)$ \\
Fall & & $9(19.1 \%)$ & $25(54.3 \%)$ \\
Motor Vehicle Accident & & $18(38.3 \%$ & $8(13.5 \%)$ \\
Sport & & $8(17 \%)$ & $10(19.2 \%)$ \\
Other & & $9(19.1 \%)$ & $1(1.9 \%)$ \\
Unknown & & $0(0 \%)$ & \\
\hline
\end{tabular}

Means and standard deviations are presented except for days since injury- presented as median with $1^{\text {st }}$ and $3^{\text {rd }}$ quartiles and injury mechanism - presented as number and percent.; NSI - Neurobehavioral Symptom Inventory

\section{Protocol and Data Collection}

All participants completed neurocognitive assessments, as well as single task (ST) and DT gait assessments at either OHSU or the VA. Participant demographic details and symptom 
severity - assessed using the neurobehavioral symptom inventory - were collected for all participants. For the mTBI populations, the number of days post-mTBI and injury mechanism were recorded.

All participants completed the ANAM, which assessed the cognitive state and psychomotor performance of individuals post-mTBI. As mentioned earlier, the test is comprised of a battery of tests that assess different aspects of neurocognition including simple reaction time, visual memory (code substitution and delayed code substitution), processing speed (procedural reaction time), working memory (mathematical processing), visuospatial ability (match to sample), and impulsivity control (Go/ No-Go). Participants performed the ANAM alone on a computer and in a quiet environment. The ANAM throughput scores, which represented a speed accuracy trade off, Go/ No-Go reaction time, Go/ No-Go commissions, and ANAM composite scores were used as the traditional cognitive computerized outcomes.

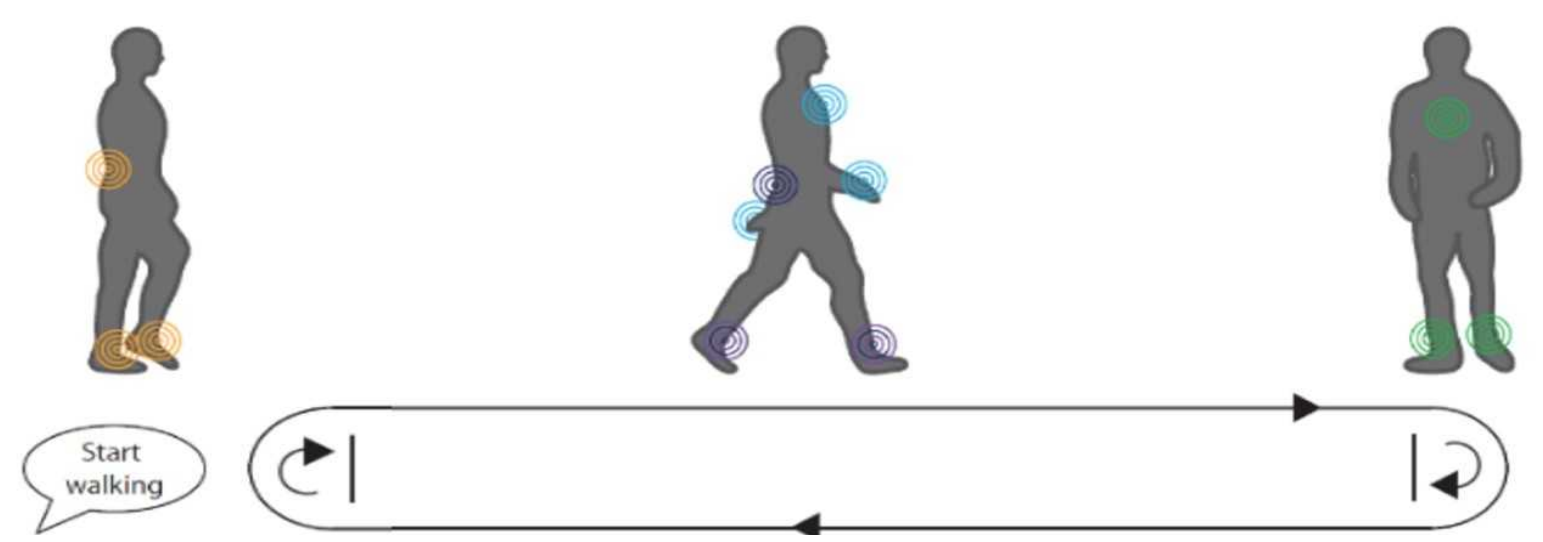

Figure 1: Schematic of a person performing the straight-line walking task and DT assessments. Participants walked back and forth turning 180 degrees at the ends of the path for the specified duration of each population (subacute $\mathrm{mTBI}=1 \mathrm{minute}$, controls and chronic mTBI $\sim 2$ minutes). Inertial sensors collected gait parameters during walking.

Following the ANAM assessment, all participants completed ST activities of a cognitive auditory Stroop task, an instrumented straight-line walking task, and the combined cognitive-motor DT gait assessment that combined these two tasks. During the cognitive auditory Stroop Test, 
participants listened to an audio recording of the words "high" or "low" spoken in high or low pitches. Participants responded with "high" or "low" based on the pitch of the voice they heard while they tried to ignore the actual spoken word. Participants sat in a quiet room listening to the auditory Stroop assessment through headphones and the percentage of correct responses was used as the outcome.

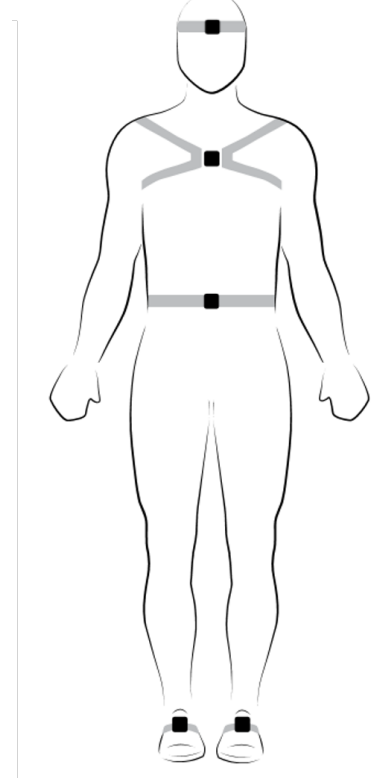

Figure 2: Inertial sensor placement for gait assessment.
For the motor task, participants walked back and forth a predetermined path at a comfortable self-selected speed (Figure 1). All participants walked for at least 1 minute over a $13 \mathrm{~m}$ distance, which has been shown as an appropriate length of time to collect and conclusively interpret steady state gait measurements (Lord et al., 2013; Kribus-Shmiel et al., 2018; Kroneberg et al., 2018). During the DT condition all participants walked and concurrently responded to the auditory Stroop task. Gait parameters were collected by 5 inertial sensors (Opals; APDM Inc., Portland, OR). The sensors were placed on the forehead, sternum, lumbar vertebrae, and on each foot (Figure 2). Over 40 gait parameters can be calculated from the sensors. However, we limited our gait outcomes to 4 parameters: double stance proportion of gait cycle variability, double stance proportion of gait cycle, stride length, and turn duration (Stuart et al., 2020). Previous research has shown that these parameters represent 4 separate domains (1. Variability - double stance proportion of gait cycle variability, 2. Rhythm - double stance proportion of gait cycle, 3. Pace - stride length, and 4. Turning - turn duration), that can characterize gait performance in non-acute mTBI, and these parameters had the 
highest loading component within each gait domain (component loadings $>0.813$ ) (Stuart et al., 2020).

\section{Statistical analysis}

Data were initially inspected for normality. Normality tests and inspection indicated a normal distribution for the ANAM composite score, ANAM subcategory throughput scores, ANAM Go/No-Go reaction time, and gait outcomes. Non-normal distribution was identified for ANAM Go/No-Go number of commissions, seated ST Stroop accuracy, and DT Stroop Accuracy. We used a one-way ANOVA to determine demographic differences between healthy control, subacute mTBI and chronic mTBI groups.

To evaluate group differences on the ANAM assessment, we fit a General Linear Model (GLM) for the ANAM composite score and each ANAM subcategory throughput score. Each GLM had a fixed factor of group with three levels: healthy control, subacute mTBI, and chronic mTBI. The healthy control group served as the reference condition. We used Tukey's HSD for post hoc pairwise comparisons. Kruskal Wallis-H tests were used to determine group effects on the outcomes of ANAM Go/No-Go number of commissions, seated ST Stroop accuracy, and DT Stroop Accuracy. Post-hoc pairwise comparisons were performed with Dunn's procedure with a Bonferroni correction for multiple companions. Only the first minute of responses during the DT auditory Stroop were used from the healthy control and chronic mTBI participants in the analysis.

To assess group differences across ST and DT conditions on gait outcomes, we fit separate linear mixed effects models (LME) for each gait outcome - double stance proportion of gait cycle variability, double stance proportion of gait cycle, stride length, and turn duration. Each 
LME model contained fixed effects for group (3 levels with the healthy control group serving as the reference), task ( 2 levels - single task and dual task with the single task condition serving as the reference condition), the group $\mathrm{x}$ task interactions, covariates for any demographic characteristics identified as significantly different between groups, and random intercepts to account for with-in subject correlations. Post hoc analyses were conducted to investigate significant group $\mathrm{x}$ condition interactions using Tukey-Kramer adjusted $p$-values to account for multiple comparisons. Statistical significance for the GLM and LME models were set to $p<0.05$. The GLM and LME model analyses were performed in SAS (v9.4, Cary, NC) and Kruskal Wallis-H tests were performed in SPSS Version 27 (IBM Corp).

\section{Results}

\section{Traditional Neuropsychological Testing Between Group Differences}

There was a significant effect of group on the ANAM composite scores $\left(F_{2,153}=12.40, p\right.$ $<0.001)$. Post hoc comparisons showed that the healthy control group had better ANAM composite scores relative to the subacute (mean difference [95\% confidence interval]: 0.776 [0.298, $1.253])$ and chronic (0.916 [0.439, 1.394]; Figure 3) mTBI groups. No differences existed between the subacute and chronic mTBI groups for the ANAM composite score $(0.141[-0.363$, $0.644])$.

Similar groups differences existed for the ANAM subcategories. The group effect was significant for the ANAM simple reaction time $\left(F_{2,153}=7.39, p<0.001\right)$, code substitution $\left(F_{2,153}\right.$ $=4.41, p=0.014)$, procedural reaction time $\left(F_{2,153}=11.89, p<0.001\right)$, mathematical processing $\left(F_{2,152}=3.22, p=0.043\right)$, match-to-sample $\left(F_{2,153}=3.85, p=0.023\right)$, delayed code substitution $\left(F_{2,153}=3.07, p=0.049\right)$, and repeated simple reaction time $\left(F_{2,153}=10.01, p<0.001\right)$ through- 


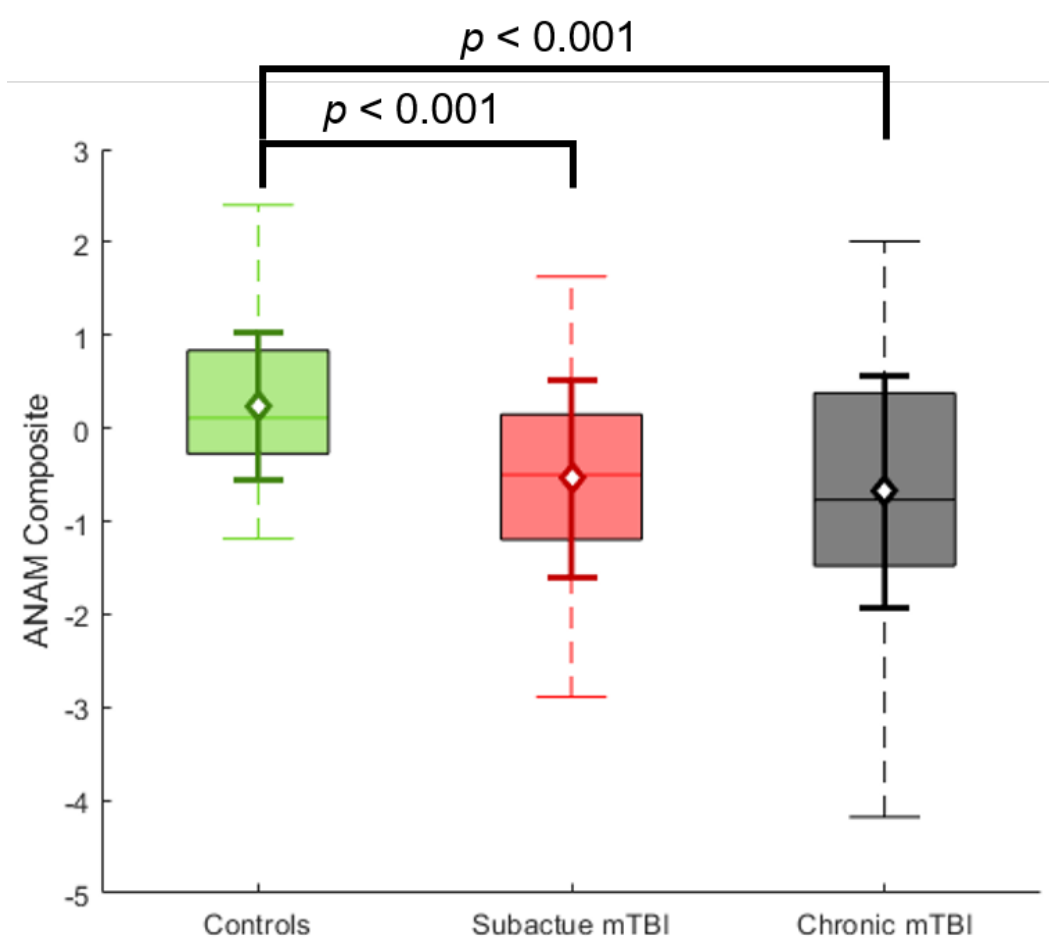

Figure 3: Box and whisker plots with means and standard deviations for ANAM composite scores for controls (green), subacute mild traumatic brain injury (mTBI - red), and chronic mTBI (black) groups.

put scores. In general, post hoc comparisons showed that the healthy control group had significantly better throughput scores relative to both subacute and chronic mTBI groups for simple reaction time, procedural reaction time, code substitution, and repeated simple reaction time subcategories (Table 2). The healthy control group had significantly better mathematical processing and delayed code substitution throughput scores relative to the subacute mTBI group (Table 2). Additionally, the healthy control group had significantly better match-to-sample throughout scores relative to the chronic mTBI group (Table 2). No significant differences existed between subacute and chronic mTBI groups on the ANAM subcategories ( $p$ 's $>0.514)$. There was no group effect on ANAM Go/No-Go reaction times $\left(F_{2,153}=0.73, p=0.485\right)$. However, there were significant group differences in the distribution of the number of Go/No-Go commissions $\left(\chi^{2}(2)\right.$ $=17.06, p<0.001$ ), where the mean rank for the number of Go/No-Go commissions for the 
healthy control group (60.38) was significantly lower and better relative to the subacute mTBI group (95.83; $\left.p_{a d j}<0.001\right)$.

Table 2: ANAM subcategory means (95\% confidence intervals) for healthy control, subacute mild traumatic brain injury (mTBI) and chronic mTBI groups. ANAM subcategory means are presented as throughput scores except for Go/No-Go reaction time.

\begin{tabular}{lccc}
\hline ANAM Subcategory & Healthy Control & Subacute mTBI & Chronic mTBI \\
\hline Simple Reaction Time & 219.00 & $193.55^{\mathrm{a}}$ & $195.34 \mathrm{a}$ \\
& $(209.09,228.91)$ & $(182.45,204.65)$ & $(184.24,206.44)$ \\
Code Substitution & 54.80 & $48.72^{\mathrm{a}}$ & $48.32^{\mathrm{a}}$ \\
& $(51.52,58.07)$ & $(45.05,52.39)$ & $(44.65,51.90)$ \\
Procedural Reaction Time & 104.22 & $89.17^{\mathrm{a}}$ & $88.92^{\mathrm{a}}$ \\
& $(99.40,109.04)$ & $(83.77,94.57)$ & $(83.51,94.32)$ \\
Mathematical Processing & 25.46 & $21.94 \mathrm{a}$ & 22.80 \\
& $(23.53,27.39)$ & $(19.77,24.10)$ & $(20.62,24.99)$ \\
Match-to-Sample & 35.33 & 31.26 & $29.19 \mathrm{a}$ \\
& $(32.32,38.34)$ & $(27.93,35.60)$ & $(25.85,32.53)$ \\
Delayed Code Substitution & 45.56 & $39.16^{\mathrm{a}}$ & 41.52 \\
& $(42.46,50.66)$ & $(34.67,43.66)$ & $(36.98,40.07)$ \\
Repeated Simple Reaction & 220.58 & $197.87^{\mathrm{a}}$ & $189.36^{\mathrm{a}}$ \\
Time & $(210.95,230.21)$ & $(187.08,208.66)$ & $(178.57,200.51)$ \\
Go/No-Go Reaction Time (ms) & 342.46 & 349.47 & 352.06 \\
& $(331.46,353.45)$ & $(337.15,361.79)$ & $(339.74,364.38)$ \\
\hline
\end{tabular}

$\mathrm{a}$ - denotes a significant difference relative to healthy controls

\section{Single Task and Dual Task Test Cognitive Group Differences}

There were significant group differences in the distributions of seated ST auditory Stroop accuracy $\left(\chi^{2}(2)=10.37, p=0.006\right)$. Specifically, the mean rank for the seated ST Stoop accuracy for the chronic mTBI group (65.68) was significantly lower and worse relative to both the healthy control group $\left(80.59 ; p_{a d j}=0.028\right)$ and the subacute $\mathrm{mTBI}$ group $\left(83.81 ; p_{a d j}=0.008\right)$. 
There was no difference between the healthy control group and subacute mTBI group $\left(p_{a d j}=\right.$ 1.00). Additionally, there were significant group differences in the distributions of DT Stroop accuracy $\left(\chi^{2}(2)=11.88, p=0.003\right)$. The mean rank for the DT Stroop accuracy for the subacute mTBI group (91.77) was significantly higher and better relative to both the healthy control group $\left(73.99 ; p_{a d j}=0.044\right)$ and the chronic mTBI group $\left(66.01 ; p_{a d j}=0.002\right)$. There was no difference between the healthy control and chronic mTBI group on DT Stroop accuracy distributions $\left(p_{a d j}=\right.$ $0.818)$.

\section{Gait}

Prior to implementing the LME models, a one-way ANOVA showed that the chronic mTBI group weighed more relative to the subacute $\mathrm{mTBI}$ group $($ mean difference $=10.35 \mathrm{~kg}$ $\left.[1.70 \mathrm{~kg}, 19.00 \mathrm{~kg}] ; p_{a d j}=0.015\right)$. For this reason and the known relationship of height and mass on gait, body mass index (BMI) was calculated for each participant and used as a covariate in the LME models. The results for fixed effects and interactions of the LME models are presented after controlling for BMI.

There was no significant interaction (group $\mathrm{x}$ task) effect on the proportion spent in double support variability $\left(F_{2,153}=0.40, p=0.6678\right)$. On average, all participants had less variability of time spent in double support stance during the DT relative to the ST $\left(F_{1,153}=4.77, p=0.0304\right.$;

A

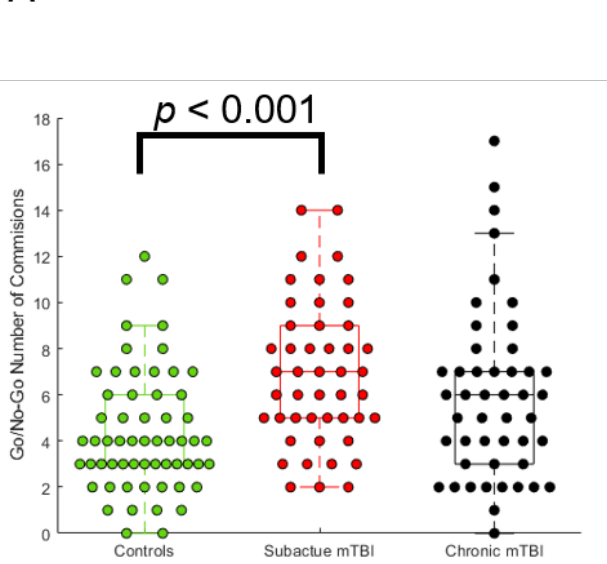

B

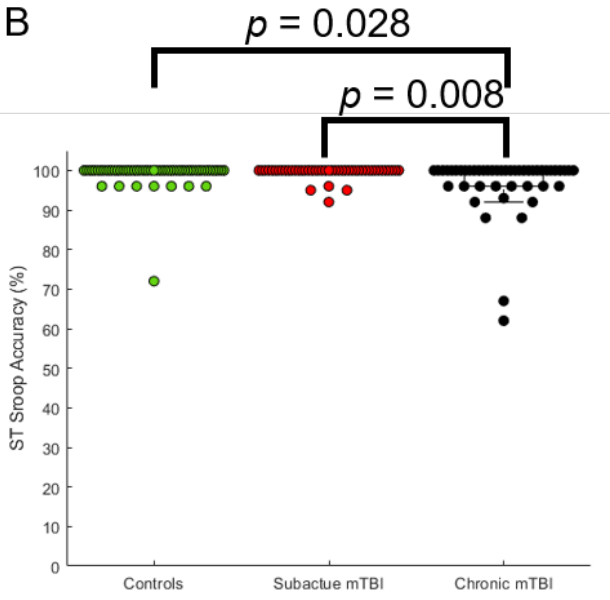

C

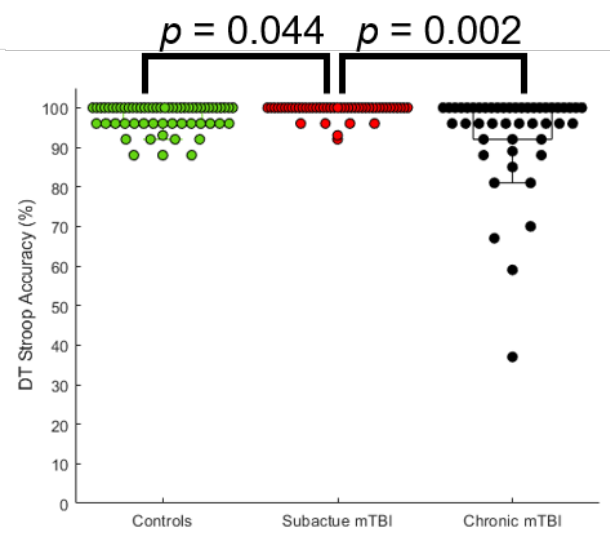

Figure 4: Distributions for non-normal cognitive data: A) Go/No-Go commissions, B) Single Task (ST) Stroop Accuracy, and C) Dual Task (DT) Stroop Accuracy for healthy control (green), subacute mild traumatic brain injury (mTBI; red), and chronic mTBI (black) groups. 
combined groups column of Table 3). There was a significant effect of group on variability of time spent in double support stance $\left(F_{2,153}=3.17, p<0.0446\right)$, where the subacute mTBI participants had significantly more double support variability across both tasks relative to healthy control participants $\left(t_{1,151}=2.50, p_{a d j}=0.0360\right.$; combined tasks row Table 3$)$.

Additionally, there was no significant interaction (group $\mathrm{x}$ task) effect on the proportion of time in double support throughout the gait cycle $\left(F_{2,153}=1.83, p=0.1636\right)$. All participants spent a larger proportion of the gait cycle in double support during the DT relative to the ST $\left(F_{1,153}=29.99, p<0.0001\right.$; combined groups column of Table 3$)$. There was a significant group effect $\left(F_{2,153}=7.02, p=0.0012\right)$ on proportion of the gait cycle in double support. Specifically, participants with chronic mTBI had a significantly larger proportion of the gait cycle spent in double support relative to healthy control $\left(t_{1,153}=3.69, p_{a d j}=0.0009\right)$ and subacute participants $\left(t_{1,153}=2.60, p_{a d j}=0.0278 ;\right.$ combined tasks row Table 3$)$.

There was no significant interaction (group $\mathrm{x}$ task) effect on participant stride length $\left(F_{2,153}=0.33, p=0.7175\right)$. All participants had a significantly shorter stride length during the DT activity relative to the $\mathrm{ST}\left(F_{1,153}=35.74, p<0.0001\right.$; combined groups column of Table 3$)$. There was a significant group effect $\left(F_{2,153}=9.64, p=0.0001\right)$ on stride length as well. Specifically, participants with chronic mTBI had a significantly shorter stride length relative to healthy con$\operatorname{trol}\left(t_{1,153}=3.83, p_{a d j}=0.0005\right)$ and subacute participants $\left(t_{1,153}=3.90, p_{a d j}=0.0004 ;\right.$ combined tasks row Table 3) regardless of task.

Finally, there was a significant interaction (group $\mathrm{x}$ task) effect on the turn duration $\left(F_{2,153}\right.$ $=3.84, p=0.0236$ ). Specifically, participants with chronic mTBI had significantly longer turn durations during the ST condition relative to the healthy control $\left(t_{1,153}=3.48, p_{a d j}=0.0019\right)$ and 
subacute participants $\left(t_{1,153}=4.98, p_{a d j}<0.0001\right)$, and had significantly longer turn durations during the DT condition relative to the healthy control $\left(t_{1,153}=5.00, p_{a d j}<0.0001\right)$ and subacute participants $\left(t_{1,153}=5.59, p_{a d j}<0.0001\right.$; Table 3$)$. There were no differences between healthy control and subacute participants within ST and DT conditions ( $\mathrm{p}_{\text {adj }}$ 's $>0.1650$ ).

Table 3: Gait outcome means (95\% confidence intervals) for each group (healthy controls, subacute mTBI, and chronic $\mathrm{mTBI}$ ) and combined groups by single task (ST), dual task (DT), and combined tasks after adjusting for body mass index.

\begin{tabular}{|c|c|c|c|c|}
\hline $\begin{array}{l}\text { Gait Outcome Accord- } \\
\text { ing to Task }\end{array}$ & $\begin{array}{l}\text { Healthy Con- } \\
\text { trol }\end{array}$ & $\begin{array}{l}\text { Subacute } \\
\text { mTBI }\end{array}$ & Chronic mTBI & $\begin{array}{l}\text { Combined } \\
\text { Groups }\end{array}$ \\
\hline \multicolumn{5}{|c|}{ Proportion in Double Support Variability } \\
\hline ST & $\begin{array}{c}0.973 \\
(0.906,1.039)\end{array}$ & $\begin{array}{c}1.095 \\
(1.021,1.169)\end{array}$ & $\begin{array}{c}1.026 \\
(0.951,1.101)\end{array}$ & $\begin{array}{c}1.031 \\
(0.990,1.072)\end{array}$ \\
\hline DT & $\begin{array}{c}0.934 \\
(0.867,1.001\end{array}$ & $\begin{array}{c}1.041 \\
(0.967,1.114)\end{array}$ & $\begin{array}{c}1.009 \\
(0.934,1.084)\end{array}$ & $\begin{array}{c}0.995^{*} \\
(0.990,1.072)\end{array}$ \\
\hline Combined Tasks & $\begin{array}{c}0.953 \\
(0.892,1.014)\end{array}$ & $\begin{array}{c}1.068 \mathrm{e} \\
(1.001,1.135)\end{array}$ & $\begin{array}{c}1.018 \\
(0.949,1.087)\end{array}$ & \\
\hline
\end{tabular}

Proportion in Double Support (\%)

$\begin{array}{lcccc}\text { ST } & 19.103 & 19.432 & 20.593 & 19.710 \\ & (18.499, & (18.767, & (19.910, & (19.338, \\ & 19.707) & 20.098) & 21.277) & 20.081) \\ \text { DT } & 19.350 & 19.867 & 21.203 & 20.140^{*} \\ & (18.745, & (19.204, & (20.519, & (19.767, \\ & 19.954) & 20.098) & 21.866) & 20.512) \\ \text { Combined Tasks } & 19.226 & 19.651 & 20.880^{c, d} & \\ & (18.635, & (19.000, & (20.229, & \\ & 19.818) & 20.301) & 21.567) & \end{array}$

Stride Length (m)
ST
1.305
1.315
1.218
1.279
(1.274, 1.335)
(1.281, 1.348)
(1.184, 1.253)
(1.260, 1.298)
DT
1.289
1.293
1.200
$1.261^{*}$
(1.259, 1.320)
$(1.260,1.326)$
$(1.165,1.234)$
$(1.242,1.279)$ 
Combined Tasks $\quad 1.297 \quad 1.304 \quad 1.209 \mathrm{c}, \mathrm{d}$

$(1.267,1.327) \quad(1.271,1.337)(1.175,1.243)$

Turn Duration (s)

$\begin{array}{lcccc}\text { ST } & 2.059 & 1.942 & 2.288^{\mathrm{a}, \mathrm{b}} & 2.096 \\ & (1.973,2.145) & (1.847,2.036) & (2.191,2.385) & (2.043,2.149) \\ \text { DT } & 2.056 & 1.997 \mathrm{c} & 2.385^{\mathrm{a}, \mathrm{b}, \mathrm{c}} & 2.146 \\ & (1.970,2.142) & (1.903,2.091) & (2.288,2.482) & (2.094,2.199) \\ \text { Combined Tasks } & 2.058 & 1.969 & 2.337 & \\ & (1.975,2.140) & (1.879,2.060) & (2.243,2.430) & \end{array}$

$\mathrm{a}$ - denotes a significant difference relative to the healthy control group within task type; $\mathrm{b}$ - denotes a significant difference relative to subacute mTBI group within task type; $\mathrm{c}$ - denotes a significant difference relative to the healthy control group for combined tasks; e - denotes a significant difference relative to the healthy controll group for combined tasks; * - denotes a significant difference relative to single task.

\section{Discussion}

The most important finding from this study is that people with subacute and chronic symptoms of mTBI demonstrate cognitive deficits, as assessed by the ANAM, weeks to even years after the initial injury. For our auditory Stroop task, both ST and DT assessments produced mixed results. The chronic mTBI group performed significantly worse than the subacute and control groups in the ST seated assessment, meaning that they responded with more errors on average than the other groups. The subacute mTBI group outperformed both the healthy control and chronic mTBI groups in the DT auditory Stroop task. This help supports that there are lingering cognitive deficits relative to healthy individuals that are still present for people with chronic symptoms of mTBI. While DT conditions had a significant effect on all groups' performance in proportion of gait cycle spent in double stance and stride length, the chronic mTBI population consistently demonstrated a more conservative gait pattern regardless of task type. 
The results of this study emphasize the importance of comprehensive neuropsychological assessments in cases of mTBI, utilizing both traditional computerized tests such as the ANAM, and DT motor-cognitive tests. By evaluating cognitive function in relation to motor function, we are further able to assess the recovery process of cognition at multiple points in time. This is vital, seeing as our results have indicated prolonged cognitive and functional deficits in the chronic mTBI population, who are often thought to be recovered by the time that they are $>3$ months post injury (Karr et al., 2014, Kwok et al., 2008, \& Levin et al.,1987, 1991). Additionally, this study has confirmed the presence of cognitive deficits in the subacute mTBI population as well. Future research in this area should further evaluate these cognitive and gait deficits in order to determine the average amount of time it takes individuals with mTBI to no longer present with symptoms.

The rationale behind conducting this study comes from the conflicting evidence of cognitive deficit presence in the months following mTBI (Dean \& Sterr, 2013). This could be due to the variability in how the field assesses cognitive deficits: computerized seated tests, or more functional motor-cognitive tests. We therefore set out to examine differences in cognitive abilities between healthy controls and people with mTBI using traditional standardized computer cognitive testing (ANAM), and DT motor-cognitive tasks. The extent to which cognitive deficits are still prevalent in chronic mTBI populations is also disputed due to conflicting evidence on when neurological symptoms are thought to resolve (Broglio et al, 2009 \& Tellier et al., 2009). The mTBI participants included in this study were at varying timepoints post-injury in order to gain a more nuanced understanding of cognitive function and gait differences during dual task assessments in relation to time since injury. 
In general both mTBI groups displayed cognitive deficits relative to healthy controls in simple reaction time, visual memory (code substitution and delayed code substitution), processing speed (procedural reaction time), working memory (mathematical processing), visuospatial ability (match to sample), and impulsivity control (Go/ No-Go number of commissions) after mTBI. Evidence suggests a positive correlation between high mTBI symptomology (determined by the Glasgow Coma Scale) and poor performance on the ANAM, when investigating people < one month from their mTBI (Sours et al., 2015). Thus, the high symptom severities reported for our subacute and chronic mTBI groups in this study could be related to the lowered cognitive function. Although this study was not intending to establish relationships between mTBI symptom severity with cognitive function, future studies could investigate this relationship.

Using other traditional neuropsychological tests that assess different cognitive processes than the ANAM, evidence has also suggested deficits in episodic memory (personal experience recall), immediate and delayed verbal memory recall, verbal recognition, general recall, object recognition, verbal fluency, and information processing speed up to 19 months after mTBI (Miotto et al., 2010, Mathias \& Wheaton, 2007, \& O'Jile et al., 2006). Due to our findings and additional evidence that physiological symptom resolution predates neurocognitive symptoms, the ANAM may be a useful tool in evaluating cognitive deficits from mTBI in both subacute and chronic populations (Porter, S.J. \& Johnson, D.E., 2020).

People experiencing mTBI symptoms more sub-acutely may not have similar levels of cognitive deficits in inhibiting a predetermined response and selecting the relevant sensory information to make the correct response (i.e. suppressing the natural response of "high" when the word high is spoken in a low pitch). The Stroop task has traditionally shown differences between 
healthy controls and people with mTBI but previous research has more often involved more visual Stroop tasks as opposed to auditory Stroop. Therefore, the seated auditory Stroop task may not be detecting cognitive changes following mTBI relative to healthy controls. In the DT assessment, the subacute mTBI group performed significantly better (higher response accuracy rate) than both the control group and the chronic group, which was unexpected. Methodological differences in the way that the groups were assessed may have influenced this result. Specifically, the subacute mTBI group had the opportunity to perform the DT auditory Stroop Test during a custom clinical turns course before performing the auditory Stroop Test during the DT straightline walking task. This additional trial performing the Auditory Stroop during a DT condition may have produced a learning effect. Additionally, response time was not recorded during seated ST and DT Stroop assessments. This may have given us a more accurate metric of DT effects than accuracy of responses, and has been used in the past when assessing DT deficits (Lee et al., 2012).

From a gait perspective, DT conditions had a significant effect on all groups' performance in regards to proportion of gait cycle spend in double stance and stride length, and the chronic mTBI population had a more conservative gait pattern regardless of task type (ST or DT). This indicates that individuals with chronic mTBI symptoms have movement deficits when walking regardless of whether they are performing a ST or DT activity. Similar results have been found in regards to mTBI populations exhibiting a slower gait speed than healthy controls for up to two months post injury (Büttner et. al, 2020). One of the novel aspects of our study is that we used parameters that characterize different aspects of gait (Rhythm, Variability, Pace, and Turning) rather than focusing only on speed. This choice was made due to previous research showing 
these parameters are independent from one another and are better able to characterize gait profile in people with mTBI (Stuart et al., 2020).

\section{Limitations}

One limitation of this study is that we did not control for subjects' prior mTBI, which may have influenced overall cognitive function. However, self-reported mTBI history can be difficult to capture because a number of potential mTBI can go undiagnosed (McCrea et al., 2015). We are limited in the conclusions we can make on cognitive deficits and recovery following mTBI in our mTBI groups with longer symptom durations because of the cross-sectional study design. Another limitation is that due to the fact that this is a secondary analysis, the data collection sessions were structured slightly differently between mTBI- although the outcome measures were identical. Our study captured people at various timepoints after injury. Future studies could employ a longitudinal design to characterize change in cognitive and motor deficits over time following mTBI.

\section{Conclusion}

All in all, cognitive testing is a vital component of mTBI evaluations and should be used in comprehensive clinical assessments. When used in conjunction with other clinical metrics of appraisal, traditional neuropsychological exams and motor-cognitive assessments can indicate the presence of neurological deficits not otherwise detected by physiological clinical exams. This is true for both the subacute and chronic mTBI populations. 


\section{Appendix A}

\section{Traditional Neuropsychological Testing Between Group Differences}
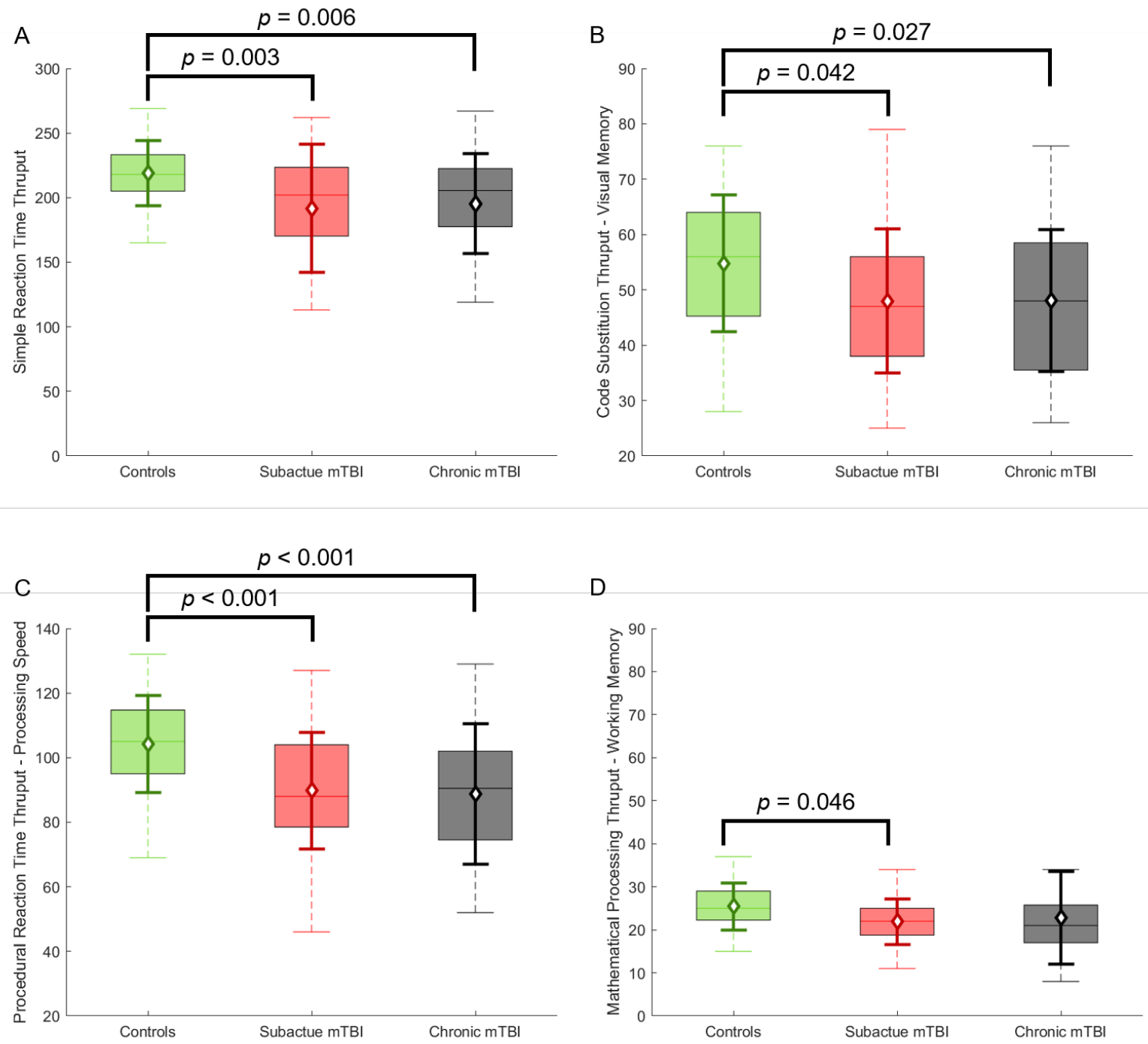

Figure A1: Box and whisker plots with means and standard deviations for ANAM A) simple reaction time, B) code substitution, C) procedural reaction time, and D) mathematical processing throughput scores for healthy controls (green), subacute mild traumatic brain injury (mTBI), and chronic mTBI groups. 

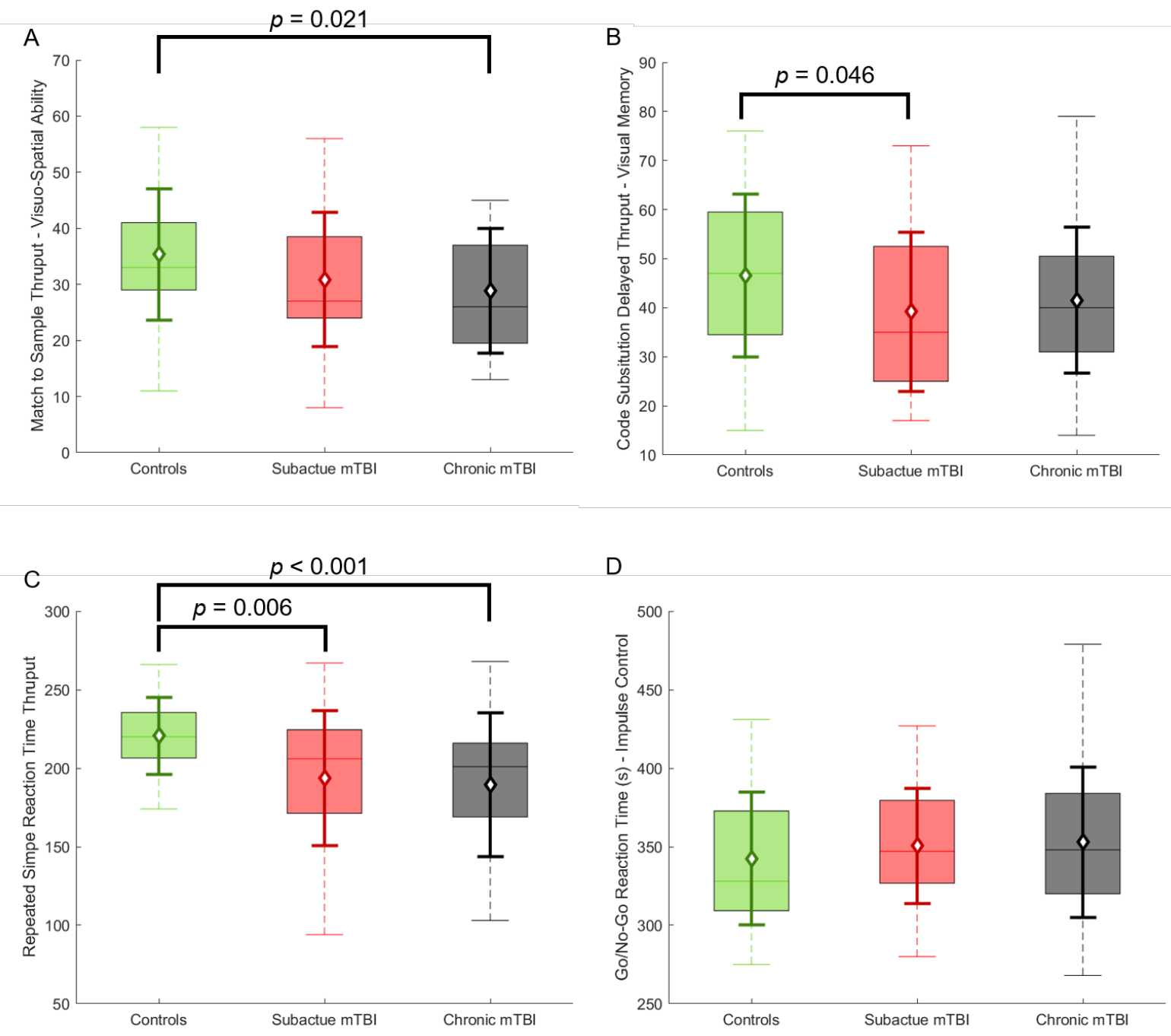

$\mathrm{D}$

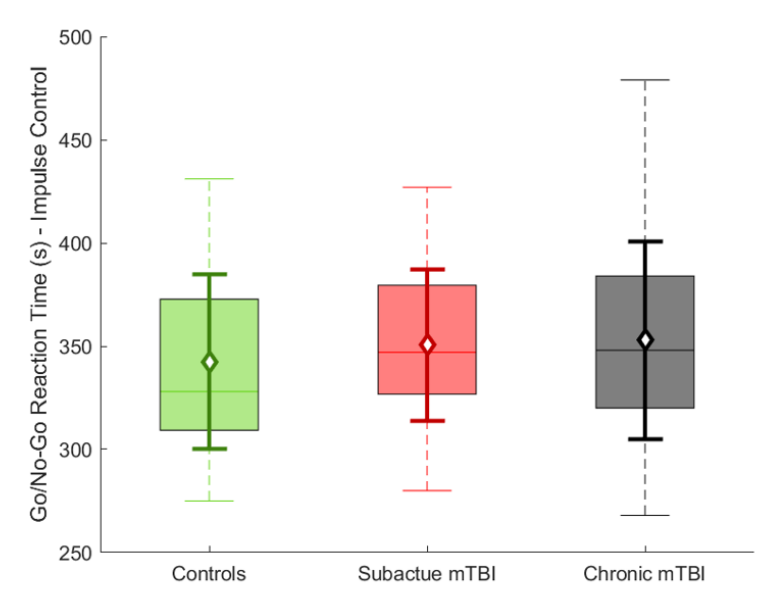

Figure A2: Box and whisker plots with means and standard deviations for ANAM A) match to sample, B) code substitution delayed, and C) repeated simple reaction time throughput scores and D) Go/No-Go reaction time for healthy controls (green), subacute mild traumatic brain injury (mTBI), and chronic mTBI groups. 


\section{References}

ANAM. (n.d.). Retrieved January 26, 2021, from http://www.vistalifesciences.com/anam-intro

Asken, B.M., Bauer, R.M., Guskiewicz, K.M., et al (2018). Immediate removal from activity after sport-related concussion is associated with shorter clinical recovery and less severe symptoms in collegiate student-athletes. Am J Sports Med, 46(6), $1465-1474$.

Avedesian, J. M., Covassin, T., Baez, S., Nash, J., Nagelhout, E., \& Dufek, J. S. (2021). Relationship between cognitive performance and lower extremity biomechanics: Implications for sports-related concussion. Orthopaedic Journal of Sports Medicine, 9(8), 23259671211032246-23259671211032246. https://doi.org/

$10.1177 / 23259671211032246$

Broglio, S.P., McAllister, T., Katz, B.P., LaPradd, M., Zhou, W., McCrea, M.A. (2021). The natural history of sport-related concussion in collegiate athletes: Findings from the NCAA-DoD CARE Consortium. Sports Med.

Broglio, S. P., Pontifex, M. B., O’Connor, P., and Hillman, C. H. (2009). The persistent effects of concussion on neuroelectric indices of attention. J. Neurotrauma 26, 1463-1470.

Büttner, F., Howell, D. R., Ardern, C. L., Doherty, C., Blake, C., Ryan, J., Catena, R., Chou, L.S., Fino, P., Rochefort, C., Sveistrup, H., Parker, T., \& Delahunt, E. (2020). Concussed athletes walk slower than non-concussed athletes during cognitive-motor dual-task assessments but not during single-task assessments 2 months after sports concussion: A systematic review and meta-analysis using individual participant 
data. British Journal of Sports Medicine, 54(2), 94-101. https://doi.org/10.1136/

bjsports-2018-100164

Coldren, R. L., Russell, M. L., Parish, R. V., Dretsch, M., \& Kelly, M. P. (2012). The ANAM lacks utility as a diagnostic or screening tool for concussion more than 10 days following injury. Military Medicine, 177(2), 179-183. https://doi.org/10.7205/ MILMED-D-11-00278

Czerniak, L. L., Liebel, S. W., Garcia, G.-G. P., Lavieri, M. S., McCrea, M. A., McAllister, T. W., \& Broglio, S. P. (2020). Sensitivity and specificity of computer-based neurocognitive tests in sport-related concussion: Findings from the NCAA-DoD CARE consortium. Sports Medicine (Auckland). https://doi.org/10.1007/s40279-020-01393$\underline{7}$

Dean, P.J., Sterr, A., (2013). Long-term effects of mild traumatic brain injury on cognitive performance. Front Hum Neurosci; 7:30.

Dretsch, M., Parish, R., Kelly, M., Coldren, R., \& Russell, M. (2015). Eight-day temporal stability of the automated neuropsychological assessment metric (ANAM) in a Deployment Environment. Applied Neuropsychology: Adult, 22(4), 304-310. https:// doi.org/10.1080/23279095.2014.926454

Fino, P. C., Peterka, R. J., Hullar, T. E., Murchison, C., Horak, F. B., Chesnutt, J. C., \& King, L. A. (2017). Assessment and rehabilitation of central sensory impairments for balance in mTBI using auditory biofeedback: A randomized clinical trial. $\mathrm{BMCNeu-}$ rology, 17(1), 41. https://doi.org/10.1186/s12883-017-0812-7 
Kwok, F.Y., Lee, T.M.C., Leung, C.H.S., \& Poon, W.S., (2008). Changes of cognitive functioning following mild traumatic brain injury over a 3-month period, Brain Injury, 22:10, 740-751, DOI: 10.1080/02699050802336989.

Howell, D., Osternig, L., Van Donkelaar, P., Mayr, U., \& Chou, L.-S. (2013). Effects of concussion on attention and executive function in adolescents. Medicine \& Science in Sports \& Exercise, 45(6), 1030-1037. https://doi.org/10.1249/ $\underline{\text { MSS.0b013e3182814595 }}$

Kamins, J., Bigler, E., Covassin, T., et al (2017). What is the physiological time to recovery after concussion? Systematic review. Br J Sports Med. bjsports-2016-097464.

Karr, J. E., Areshenkoff, C. N., \& Garcia-Barrera, M. A. (2014). The Neuropsychological Outcomes of Concussion: A Systematic Review of Meta-Analyses on the Cognitive Sequelae of Mild Traumatic Brain Injury. Neuropsychology, 28(3), 321-336. https://doi.org/10.1037/neu0000037

Kleiner, M., Wong, L., Dubé, A., Wnuk, K., Hunter, S. W., \& Graham, L. J. (2017). Dual-task assessment protocols in concussion assessment: A systematic literature review. Journal of Orthopaedic \& Sports Physical Therapy, 48(2), 87-103. https:// doi.org/10.2519/jospt.2018.7432

Lee, H., Sullivan, S. J., \& Schneiders, A. G. (2012). The use of the dual-task paradigm in detecting gait performance deficits following a sports-related concussion: A systematic review and meta-analysis. Journal of Science and Medicine in Sport, 16(1), 2-7. https://doi.org/10.1016/j.jsams.2012.03.013 
Levin, H.S., Amparo, E., Eisenberg, H.M., Williams, D.H., High, W.M., McArdle, C.B., \& Weiner, R.L. (1987). Magnetic resonance imaging and computerized tomography in relation to the neurobehavioral sequelae of mild and moderate head injuries. Journal of Neurosurgery, 66, 706-713.

Levin, H.S., Williams, D.H., Eisenberg, H.M., High, W.M., \& Guinto, F.C. (1991). Serial MRI and neurobehavioural findings after mild to moderate closed head injury. Journal of Neurology, Neurosurgery, and Psychiatry, 55, 255-262.

Mathias, J., Wheaton, P. (2007). Changes in attention and information-processing speed following severe traumatic brain injury. Neuropsychology; 21:212-223.

Martini, D.N., Parrington L., Stuart, S., Fino, P.C., King, L.A (2021). Gait performance in people with symptomatic, chronic mild traumatic brain injury. J Neurotrauma, 38(2), 218-224.

Maruta, J., Spielman, L. A., Yarusi, B. B., Wang, Y., Silver, J. M., \& Ghajar, J. (2016). Chronic post-concussion neurocognitive deficits. II. Relationship with Persistent Symptoms. Frontiers in Human Neuroscience. http://dx.doi.org.proxy.lib.pdx.edu/ $\underline{10.3389 / \text { fnhum.2016.00045 }}$

McCrea, M., Broshek, D.K., \& Barth, J.T., (2015). Sports concussion assessment and management: Future research directions, Brain Injury, 29:2, 276-282, DOI: $\underline{10.3109 / 02699052.2014 .965216}$

McCrea, M., Guskiewicz, K. M., Marshall, S. W., Barr, W., Randolph, C., Cantu, R. C., Onate, J. A., Yang, J., \& Kelly, J. P. (2003). Acute effects and recovery time following con- 
cussion in collegiate football players: The NCAA concussion study. JAMA, 290(19), 2556. https://doi.org/10.1001/jama.290.19.2556

McCrory, P., Meeuwisse, W., Dvorak, J., Aubry, M., Bailes, J., Broglio, S., Cantu, R. C., Cassidy, D., Echemendia, R. J., Castellani, R. J., Davis, G. A., Ellenbogen, R., Emery, C., Engebretsen, L., Feddermann-Demont, N., Giza, C. C., Guskiewicz, K. M., Herring, S., Iverson, G. L., ... Vos, P. E. (2017). Consensus statement on concussion in sport-The 5th international conference on concussion in sport held in Berlin, October 2016. British Journal of Sports Medicine, 51(11), 838-847. https:// doi.org/10.1136/bjsports-2017-097699

McInnes, K., Friesen, C.L., MacKenzie, D.E., Westwood, D.A., Boe, S.G. (2017). Mild Traumatic Brain Injury (mTBI) and chronic cognitive impairment: A scoping review. PLoS ONE, 12(4): e0174847. https://doi.org/10.1371/journal.pone.0174847

Miotto, E. C., Cinalli, F. Z., Serrao, V. T., Benute, G. G., Lucia, M. C., \& Scaff, M. (2010). Cognitive deficits in patients with mild to moderate traumatic brain injury. Arquivos de Neuro-Psiquiatria, 68, 862-868.

Nelson, L. D., Temkin, N. R., Dikmen, S., Barber, J., Giacino, J. T., Yuh, E., Levin, H. S., McCrea, M. A., Stein, M. B., Mukherjee, P., Okonkwo, D. O., Robertson, C. S., Diaz-Arrastia, R., Manley, G. T., \& and the TRACK-TBI Investigators. (2019). Recovery after mild traumatic brain injury in patients presenting to US level I trauma centers: A transforming research and clinical knowledge in traumatic brain injury (TRACK-TBI) study. JAMA Neurology, 76(9), 1049-1059. https://doi.org/ $\underline{10.1001 / \text { jamaneurol.2019.1313 }}$ 
O'Jile, J.R., Ryan, L.M., Betz, B., et al. (2006). Information processing following mild head injury. Arch Clinic Neuropsychology; 21:293-296.

Parrington, L., Jehu, D. A., Fino, P. C., Stuart, S., Wilhelm, J., Pettigrew, N., Murchison, C. F., El-Gohary, M., VanDerwalker, J., Pearson, S., Hullar, T., Chesnutt, J. C., Peterka, R. J., Horak, F. B., \& King, L. A. (2020). The sensor technology and rehabilitative timing (START) protocol: A randomized controlled trial for the rehabilitation of mild traumatic brain injury. Physical Therapy, 100(4), 687-697. https://doi.org/ $10.1093 / \mathrm{ptj} / \mathrm{pzaa} 007$

Plummer, P., \& Eskes, G. (2015). Measuring treatment effects on dual-task performance: A framework for research and clinical practice. Frontiers in Human Neuroscience, 9. https://doi.org/10.3389/fnhum.2015.00225

Polinder, S., Cnossen, M.C., Real, R.G.L., Covic, A., Gorbunova, A., Voormolen, D.C., Master, C.L., Haagsma, J.A., Diaz-Arrastia, R. \& von Steinbuechel, N. (2018) A multidimensional approach to post-concussion symptoms in mild traumatic brain injury. Front. Neurol.9:1113. doi: 10.3389/fneur.2018.01113

Recovery | Concussion | Traumatic Brain Injury | CDC Injury Center. (2020, August 31). https:// www.cdc.gov/traumaticbraininjury/recovery.html

Reeves, D. L., Winter, K. P., Bleiberg, J., \& Kane, R. L. (2007). ANAM® genogram: Historical perspectives, description, and current endeavors ¿ $^{2}$. Archives of Clinical Neuropsychology, 22(Suppl_1), S15-S37. https://doi.org/10.1016/j.acn.2006.10.013

Shor, R. E. (1975). An auditory analog of the stroop test. Journal of General Psychology, 93, $281-288$. 
Sours, C., Rosenberg, J., Kane, R. et al., (2015). Associations between interhemispheric functional connectivity and the Automated Neuropsychological Assessment Metrics (ANAM) in civilian mild TBI. Brain Imaging and Behavior 9, 190-203. https:// doi.org/10.1007/s11682-014-9295-y.

Porter, S.J. \& Johnson, D.E., (2020). Clinical Use of the Automated Neuropsychological Assessment Metrics TBI-Mil Expanded Battery in Evaluating Concussion Recovery: A Retrospective Study, Military Medicine,185, 9(10), e1722-e1727, https:// doi.org/10.1093/milmed/usaa075.

Stuart, S., Parrington, L., Morris, R., Martini, D.N., Fino, P.C., King, L.A. (2020). Gait measurement in chronic mild traumatic brain injury: A model approach. Human Movement Science, 69:102557.

Tellier, A., Marshall, S. C., Wilson, K. G., Smith, A., Perugini, M., and Stiell, I. G. (2009). The heterogeneity of mild traumatic brain injury: Where do we stand? Brain Inj. 23, 879-887. 